\title{
A Generating Function for Triangular Partitions
}

\author{
By L. Carlitz* and Richard Scoville \\ To D. H. Lehmer on his seventieth birthday
}

Abstract. Let $T_{k}(n)$ denote the number of solutions in nonnegative integers $a_{i}$, of the equation

$$
n=\sum_{i=1}^{k} \sum_{j=1}^{k-i+1} a_{i j}
$$

where the $a_{i j}$ satisfy the inequalities $a_{i j} \geqslant a_{i+1, j}, a_{i j} \geqslant a_{i+1, j-1}$. We show that

$$
\sum_{n=1}^{\infty} T_{k}(n) x^{n}=(1-x)^{-k}\left(1-x^{3}\right)^{-k+1}\left(1-x^{5}\right)^{-k+2} \cdots\left(1-x^{2 k-1}\right)^{-1} \text {. }
$$

1. Introduction. We consider the triangular array of nonnegative integers $\left(a_{i j}\right)$

$$
\begin{aligned}
& a_{11} \quad a_{12} \quad a_{13} \quad \cdots \quad a_{1 k} \\
& \begin{array}{llll}
a_{21} & a_{22} & \cdots & a_{2, k-1}
\end{array} \\
& T_{k}: \quad a_{31} \quad a_{32} \quad \cdots \\
& a_{k 1}
\end{aligned}
$$

satisfying the following system of inequalities:

$$
a_{i j} \geqslant a_{i+1, j}, \quad a_{i j} \geqslant a_{i+1, j-1} .
$$

If in addition, the $a_{i j}$ satisfy

$$
\sum_{i+j \leqslant n+1} a_{i j}=n
$$

we call $T_{k}$ a triangular partition of $n$ of order $k$.

Let $T_{k}(n)$ denote the number of arrays $T_{k}$ satisfying (1.2) and (1.3). Clearly

$$
T_{k}(0)=1 \quad(k=1,2,3, \cdots) .
$$

Since

$$
T_{1}(n)=1 \quad(n=0,1,2, \cdots),
$$

Received April 5, 1974

AMS (MOS) subject classifications (1970). Primary 05A15, 05A17; Secondary 10A45.

Key words and phrases. Partitions, generating functions.

*Supported in part by NSF grant GP-17324. 
it follows at once that

$$
\sum_{n=0}^{\infty} T_{1}(n) x^{n}=\frac{1}{1-x}
$$

For $k=2$ we have

$$
\begin{aligned}
\sum_{n=0}^{\infty} T_{2}(n) x^{n} & =\sum_{n=0}^{\infty} x^{n} \sum_{a+b+c=n ; a \geqslant c, b \geqslant c} 1 \\
& =\sum_{a \geqslant b, c \geqslant b} x^{a+b+c}=\sum_{a, b, c=0}^{\infty} x^{a+b+3 c,}
\end{aligned}
$$

so that

$$
\sum_{n=0}^{\infty} T_{2}(n) x^{n}=\frac{1}{(1-x)^{2}\left(1-x^{3}\right)} .
$$

Since $(1-x)^{-2}\left(1-x^{3}\right)^{-1}=\sum_{r=0}^{\infty}(r+1) x^{r} \sum_{s=0}^{\infty} x^{3 s}$, it follows that $T_{2}(n)=\Sigma_{3 s \leqslant n}(n-3 s+1)$. Hence, if $m=[n / 3]$, we get

$$
T_{2}(n)=1 / 2(m+1)(2 n-3 m+2) \text {. }
$$

For $k=3$ we find that

$$
\sum_{n=0}^{\infty} T_{3}(n) x^{n}=(1-x)^{-3}\left(1-x^{3}\right)^{-2}\left(1-x^{5}\right)^{-1}
$$

For $k=4$ we have

$$
\sum_{n=0}^{\infty} T_{4}(n) x^{n}=(1-x)^{-4}\left(1-x^{3}\right)^{-3}\left(1-x^{5}\right)^{-2}\left(1-x^{7}\right)^{-1}
$$

The formulas (1.6), (1.7), (1.9), (1.10) suggest the general result

$$
\text { (1.11) } \sum_{n=0}^{\infty} T_{k}(n) x^{n}=(1-x)^{-k}\left(1-x^{3}\right)^{-k+1}\left(1-x^{5}\right)^{-k+2} \cdots\left(1-x^{2 k-1}\right)^{-1}
$$

The direct proof of (1.10) is rather tedious; the corresponding proof in the case $k=5$ has not been completely carried out. We shall accordingly prove the general result (1.11) by an entirely different method which makes use of known results concerning MacMahon's theorem on $k$-line partitions [4, p. 243].

Put

$$
\frac{1}{(1-x)\left(1-x^{3}\right) \cdots\left(1-x^{2 k-1}\right)}=\sum_{n=0}^{\infty} q_{k}(n) x^{n}
$$

so that $q_{k}(n)$ is the number of partitions of $n$ into the parts $1,3,5, \cdots, 2 k-1$, repetitions allowed. Then (1.11) yields the recurrence

$$
T_{k}(n)=\sum_{j=0}^{n} q_{k}(j) T_{k-1}(n-j) .
$$


This evidently implies

$$
T_{k}(n)=\sum q_{k}\left(j_{1}\right) q_{k-1}\left(j_{2}\right) \cdots q_{2}\left(j_{k-1}\right),
$$

where the summation is over all nonnegative $j_{1}, j_{2}, \cdots, j_{k-1}$ satisfying $j_{1}+j_{2}+$ $\cdots+j_{k-1} \leqslant n$.

Formulas (1.12) and (1.13) are indeed equivalent to (1.11). Thus a combinatorial proof of either (1.12) or (1.13) would yield a combinatorial proof of (1.11).

Another result equivalent to (1.11) is the following:

$$
T_{k}(n)=\sum \prod_{j=1}^{k}\left(\begin{array}{c}
k-j+n_{j}-1 \\
n_{j}
\end{array}\right),
$$

where the outer summation is over all nonnegative $n_{1}, n_{2}, \cdots, n_{k}$ satisfying $n_{1}+$ $3 n_{2}+5 n_{3}+\cdots+(2 k-1) n_{k}=n$.

2. Special Cases. We shall now sketch the proof of (1.9). To begin with, it follows from the definition that

$$
\sum_{n=0}^{\infty} T_{3}(n) x^{n}=\left(1-x^{6}\right)^{-1} \sum_{n=0}^{\infty} T_{3}^{\prime}(n) x^{n},
$$

where $T_{3}^{\prime}(n)$ denotes the number of arrays

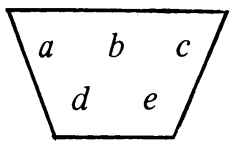

satisfying $a \geqslant d, b \geqslant d, b \geqslant e, c \geqslant e$ and $a+b+c+d+e=n$. It follows that

$$
\begin{aligned}
\sum_{n=0}^{\infty} T_{3}^{\prime}(n) x^{n} & =\sum_{d, e=0}^{\infty} x^{d+e} \sum_{a, b, c ; a \geqslant d, b \geqslant d ; b \geqslant e, c \geqslant e} x^{a+b+c} \\
& =\sum_{d, e=0}^{\infty} x^{2 d+2 e} \sum_{a, c=0}^{\infty} x^{a+c} \sum_{b \geqslant d, b \geqslant e} x^{b} \\
& =(1-x)^{-2} \sum_{b=0}^{\infty} x^{b} \sum_{d=0}^{b} \sum_{e=0}^{b} x^{2 d+2 e}=(1-x)^{-2} \sum_{b=0}^{\infty} x^{b}\left(\frac{1-x^{2 b+2}}{1-x^{2}}\right)^{2} \\
& =(1-x)^{-2}\left(1-x^{2}\right)^{-2}\left\{\frac{1}{1-x}-\frac{2 x^{2}}{1-x^{3}}+\frac{x^{4}}{1-x^{5}}\right\} \\
& =\frac{1+x^{3}}{(1-x)^{3}\left(1-x^{3}\right)\left(1-x^{5}\right)}
\end{aligned}
$$

Substituting from (2.2) in (2.1), we get (1.9).

The proof of (1.10) is a good deal more involved and we give only a brief out- 
line. To begin with, we have

$$
\sum_{n=0}^{\infty} T_{4}(n) x^{n}=\left(1-x^{10}\right)^{-1} \sum_{n=0}^{\infty} T_{4}^{\prime}(n) x^{n},
$$

where $T_{4}^{\prime}(n)$ denotes the number of arrays

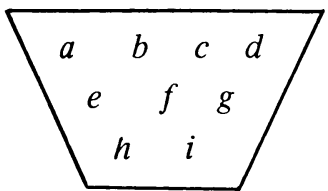

satisfying

$a \geqslant e, b \geqslant e, b \geqslant f, c \geqslant f, c \geqslant g, d \geqslant g, e \geqslant h, f \geqslant h, f \geqslant i, g \geqslant i$

and $a+b+\cdots+n+i=n$. In the next place we remove the corners on the top line of (2.4) to get

$$
\sum_{n=0}^{\infty} T_{4}^{\prime}(n) x^{n}=(1-x)^{-2} \sum x^{b+c+2 e+f+2 g+n+i},
$$

where the summation on the right is over all arrays

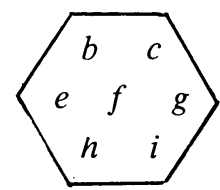

satisfying

$$
b \geqslant e, \quad b \geqslant f, \quad c \geqslant f, \quad c \geqslant g, \quad e \geqslant n, \quad f \geqslant n, \quad f \geqslant i, \quad g \geqslant i .
$$

Thus we get for the sum on the right of (2.5)

$$
\begin{array}{r}
\left(1-x^{2}\right)^{-2} \sum_{f=0}^{\infty} x^{f}\left\{\frac{x^{2 f}}{(1-x)^{2}}\left(\frac{1-x^{3 f+3}}{1-x^{3}}\right)^{2}-\frac{2 x^{4 f+2}}{(1-x)\left(1-x^{3}\right)} \frac{1-x^{3 f+3}}{1-x^{3}} \frac{1-x^{f+1}}{1-x}\right. \\
\left.+\frac{x^{6 f+4}}{\left(1-x^{3}\right)^{2}}\left(\frac{1-x^{f+1}}{1-x}\right)^{2}\right\} .
\end{array}
$$

This reduces to

$$
\left(1+x^{5}\right) /(1-x)^{2}\left(1-x^{3}\right)^{3}\left(1-x^{5}\right)\left(1-x^{7}\right) .
$$

Hence, combining (2.3), (2.5) and (2.6), we get (1.10).

3. Restatement of Problem. It will be convenient to modify the original statement of the problem. Let $A_{n}$ denote the set of lattice points in the first quadrant defined by 


$$
\mathrm{A}_{n}=\{(i, j) \mid i \geqslant 0, j \geqslant 0, i+j<n\} .
$$

$A_{n}$ is partially ordered if we put

$$
(i, j) \leqslant\left(i^{\prime}, j^{\prime}\right) \rightleftarrows i \leqslant i^{\prime} \quad \text { and } j \leqslant j^{\prime} .
$$

A nonnegative integer-valued function $f$ defined on $A_{n}$ will be called increasing if, for every $a, b \in \mathrm{A}_{n}$, we have

$$
a \leqslant b \Rightarrow f(a) \leqslant f(b) .
$$

If $f$ is increasing and takes on only the values 0 and 1 , we may associate with $f$ the subset $A_{f}$ of $A_{n}$ defined by

$$
a \in A_{f} \rightleftarrows f(a)=1 \text {. }
$$

The collection of such subsets will be denoted by $L_{n}$. Note that $L_{n}$ is a lattice with respect to union and intersection of sets. We show that $L_{n}$ contains

$$
C_{n+2}=\frac{1}{n+2}\left(\begin{array}{c}
2 n+2 \\
n+1
\end{array}\right)
$$

sets; $C_{n}$ is a so-called Catalan number (for references see [1], [3]).

If $f$ is increasing on $A_{n}$ we put

$$
\sigma(f)=\sum_{a \in A_{n}} f(a)
$$

and

$$
Q_{n}(x)=\sum x^{\sigma(f)},
$$

where the summation is over all nonnegative integer-valued increasing functions on $A_{n}$. Clearly

$$
Q_{n}(x)=\sum_{N=0}^{\infty} T_{n}(N) x^{N},
$$

where $T_{n}(N)$ is the partition function defined in the introduction.

We remark, that if we define

$$
\bar{Q}_{n}(x)=\sum x^{\sigma(f)} y^{\max f}
$$

and replace $A_{n}$ by

$$
B_{n}=\{(i, j) \mid 0 \leqslant i<n, 0 \leqslant j<n\} .
$$

then we are led to MacMahon's theorem for plane partitions.

4. The Lattice $L_{n}$. For every $A \in L_{n}$, let $g_{A}$ denote the function defined by

$$
g_{A}(i)=\operatorname{card}\left\{(n-i, j) \mid(n-i, j) \in \mathrm{A}_{n}-A\right\} \quad(i=0,1, \cdots, n) .
$$

Note that 
(i) $g_{A}$ is increasing, and

(ii) $0 \leqslant g_{A}(i) \leqslant i \quad(i=0,1, \cdots, n)$.

Moreover, if $A$ and $B$ are in $L_{n}$, then

$$
g_{A \cup B}=\min \left(g_{A}, g_{B}\right), \quad g_{A \cup B}=\max \left(g_{A}, g_{B}\right) .
$$

Let $F_{n}$ consist of all integer-valued functions satisfying (i) and (ii). Then $F_{n}$ is a lattice with respect to $\min$ and $\max$. We summarize these observations in the following theorem.

Theorem 1. The lattices $L_{n}$ and $F_{n}$ are anti-isomorphic and contain

$$
C_{n+2}=\frac{1}{n+2}\left(\begin{array}{c}
2 n+2 \\
n+1
\end{array}\right)
$$

elements.

Proof. We show first that if $f \in F_{n}$, then $f=g_{A}$ for some $A \in L_{n}$. Let $f \in F_{n}$ and put

$$
A=\{(i, j) \mid f(n-i) \leqslant j\} \cap A_{n} .
$$

Now suppose $\left(i_{0}, j_{0}\right) \in A$ and both $\left(i_{0}+1, j\right)$ and $\left(i_{0}, j_{0}+1\right) \in A_{n}$. Then

$$
f\left(n-i_{0}-1\right) \leqslant f\left(n-i_{0}\right) \leqslant j_{0}, \quad f\left(n-i_{0}\right) \leqslant j_{0}<j_{0}+1,
$$

so both $\left(i_{0}+1, j_{0}\right)$ and $\left(i_{0}, j_{0}+1\right) \in A$. Hence $A \in L_{n}$ and

$$
\begin{aligned}
g_{A}\left(n-i_{0}\right) & =\operatorname{card}\left\{j \mid\left(i_{0}, j\right) \in A_{n}-A\right\} \\
& =\operatorname{card}\left\{j \mid f\left(n-i_{0}\right)>j, j \geqslant 0\right\}=f\left(n-i_{0}\right) .
\end{aligned}
$$

This, together with the previous remarks, shows $L_{n}$ and $F_{n}$ are indeed anti-isomorphic. It is well known (see for example [3]) that the number of elements in $F_{n}$ is given by (4.3).

We note, for later use, that

$$
|A|+\sum_{i=0}^{n} g_{A}(i)=\left|A_{n}\right|=1 / 2 n(n+1) \text {. }
$$

5. Chains in $L_{n}$. By a chain in $L_{n}$ we will mean any finite or infinite sequence of sets $A_{i} \in L_{n}$ satisfying

$$
A_{i} \subseteq A_{i+1} \quad(i=0,1,2, \cdots) .
$$

We will say that the chain $\left\{A_{i}\right\}_{0}^{k}$ begins at $\phi$ and ends at $A_{n}$ if $A_{0}=\phi$ and $A_{k}=$ $A_{n}$.

There is a 1-1 correspondence between the set of increasing functions bounded by $r$ on $A_{n}$ and the chains $\left\{A_{i}\right\}_{0}^{r+1}$ in $L_{n}$ which begin at $\phi$ and end at $A_{n}$. This correspondence is given by

$$
A_{i}=\{a \mid f(a) \geqslant r-i+1\} \quad(i=0,1, \cdots, r+1) .
$$


It is clear that

$$
\sigma(f)=\left|A_{1}\right|+\left|A_{2}\right|+\cdots+\left|A_{r}\right|
$$

where

$$
\sigma(f)=\sum_{a \in A_{n}} f(a)
$$

Transferring the sets $A_{i}$ to functions in $F_{n}$ by the anti-automorphism of Theorem 1, we obtain

THEOREM 2. There is a 1-1 correspondence between the set of increasing functions bounded by $r$ on $A_{n}$ and sequences of functions $\left\{f_{i}\right\}_{0}^{r+1}$ from $F_{n}$ satisfying

$$
f_{0} \geqslant f_{1} \geqslant f_{1} \geqslant \cdots \geqslant f_{r} \geqslant 0 ; \quad f_{0}(x)=x
$$

Moreover

$$
\sigma(f)=1 / 2 m(n+1)-\sum_{i=1}^{r} \sum_{j=0}^{n} f_{i}(j) .
$$

Proof. Follows from Theorem 1 and (4.4).

Another relation between increasing functions on $A_{n}$ and chains in $L_{n}$ is given as follows. Call a chain $\left\{A_{i}\right\}$ proper if $A_{0} \neq \phi$ and $A_{i} \neq A_{i+1}$. Suppose $f$ is an increasing function on $A_{n}$ assuming the distinct nonzero values

$$
t_{1}, t_{1}+t_{2}, \cdots, t_{1}+t_{2}+\cdots+t_{j} ; \quad t_{i}>0 \text {. }
$$

Let

$$
B_{i}=\left\{a \mid f(a) \geqslant t_{1}+\cdots+t_{j-i}\right\} \quad(i=0,1, \cdots, j-1) .
$$

Then we have

$$
\sigma(f)=t_{1}\left|B_{j-1}\right|+t_{2}\left|B_{j-2}\right|+\cdots+t_{j}\left|B_{0}\right| .
$$

Hence the following theorem is immediate.

THEOREM 3. The generating function

$$
Q_{n}(x)=\sum x^{\sigma(f)} \quad\left(f \text { increasing on } A_{n}\right)
$$

is given by

$$
Q_{n}(x)=1+\sum \frac{x^{\left|B_{0}\right|}}{1-x^{\left|B_{0}\right|}} \cdots \frac{x^{\left|B_{j}\right|}}{1-x^{\left|B_{j}\right|}},
$$

where the summation is taken over all proper chains in $L_{n}$.

6. Computation of $Q_{n}(x)$. By Theorem 2 there is a 1-1 correspondence between increasing functions on $A_{n}$ bounded by $r$ and $n \times r$ arrays $\left\{f_{j}(i)\right\}$ satisfying 


$$
0 \leqslant f_{j}(1) \leqslant f_{j}(2) \leqslant \cdots \leqslant f_{j}(n) \quad(j=1,2, \cdots, r)
$$

and

$$
i \geqslant f_{1}(i) \geqslant f_{2}(i) \geqslant \cdots \geqslant f_{r}(i) \geqslant 0 \quad(i=1,2, \cdots, n) .
$$

Let $Q_{n}^{(r)}(x)$ denote the partition generating function for such arrays, that is,

$$
Q_{n}^{(r)}(x)=\sum x^{\Sigma_{i, j} f_{j}(i)},
$$

where the outer sum is taken over all $\left\{f_{j}(i)\right\}$ satisfying (6.1) and (6.2). Specializing formula (6.12) of [2], we get

$$
\begin{aligned}
Q_{n}^{(r)}(x) & =x^{1 / 2 n(n+1)}\left|x^{1 / 2(i-j)(i-j-1)}\left[\begin{array}{c}
n-j+r \\
r-i+j-1
\end{array}\right]\right| \\
& =x^{1 / 2 n(n+1)}\left|x^{1 / 2(i-j)(i-j+1)}\left[\begin{array}{c}
r+j-1 \\
2 j-i
\end{array}\right]\right| \quad(i, j=1,2, \cdots, n),
\end{aligned}
$$

where

$$
\left[\begin{array}{l}
k \\
j
\end{array}\right]=\frac{(x)_{k}}{(x)_{j}(x)_{k-j}}, \quad(x)_{k}=(1-x)\left(1-x^{2}\right) \cdots\left(1-x^{k}\right) .
$$

Replacing $x$ by $x^{-1}$, it is easily verified that

$$
\left[\begin{array}{l}
k \\
j
\end{array}\right] \rightarrow x^{j(j-k)}\left[\begin{array}{l}
k \\
j
\end{array}\right]
$$

Thus we get

$$
Q_{n}^{(r)}\left(\frac{1}{x}\right)=x^{-1 / 2 r n(n+1)}\left|x^{1 / 2(i-j)^{2}}\left[\begin{array}{c}
j+r-1 \\
2 j-i
\end{array}\right]\right| \quad(i, j=1,2, \cdots, n)
$$

By (5.6), we have

$$
Q_{n}(x)=\lim _{r \rightarrow \infty} x^{1 / 2 r n(n+1)} Q_{n}^{(r)}\left(\frac{1}{x}\right)
$$

and therefore

$$
Q_{n}(x)=\left|\frac{x^{(i-j)^{2}}}{(x)_{2 j-i}}\right|=\left|\frac{x^{(i-j)^{2}}}{(x)_{2 i-j}}\right| \quad(i, j=1,2, \cdots, n) .
$$

It is convenient to put

$$
D_{k}=\left|x^{(i-j)^{2}}\left[\begin{array}{r}
2 i \\
j
\end{array}\right]\right| \quad(i, j=1,2, \cdots, n),
$$

so that (6.6) becomes

$$
Q_{n}(x)=\frac{(x)_{1}(x)_{2} \cdots(x)_{n}}{(x)_{2}(x)_{4} \cdots(x)_{2 n}} D_{n}
$$


We shall now evaluate $D_{k}$. Let $R_{i}$ denote the $i$ th row of $D_{k}$. We shall replace $R_{k}$ by

$$
\bar{R}_{k}=R_{k}-x\left[\begin{array}{l}
k \\
1
\end{array}\right]^{\prime} R_{k-1}+x^{2}\left[\begin{array}{l}
k \\
2
\end{array}\right]^{\prime} R_{k-2}-\cdots+(-1)^{k-1} a^{k-1}\left[\begin{array}{c}
k \\
k-1
\end{array}\right]^{\prime} R_{1},
$$

where

$$
\left[\begin{array}{l}
k \\
j
\end{array}\right]^{\prime}=\frac{\left(x^{2}\right)_{k}^{\prime}}{\left(x^{2}\right)_{j}^{\prime}\left(x^{2}\right)_{k-j}^{\prime}}, \quad(a)_{k}^{\prime}=(1-a)\left(1-x^{2} a\right) \cdots\left(1-x^{2 k-2} a\right) .
$$

Then the $j$ th element in $\bar{R}_{k}$ is equal to

$$
\begin{aligned}
r_{j} & =\sum_{s=0}^{k-1}(-1)^{s} x^{s}\left[\begin{array}{l}
k \\
s
\end{array}\right]^{\prime} a^{(k-s-j)^{2}}\left[\begin{array}{c}
2 k-2 s \\
j
\end{array}\right] \\
& =\sum_{s=0}^{k}(-1)^{k-s} x^{k-s}\left[\begin{array}{l}
k \\
s
\end{array}\right]^{\prime} x^{(s-j)^{2}}\left[\begin{array}{r}
2 s \\
j
\end{array}\right] .
\end{aligned}
$$

Since

$$
\left[\begin{array}{r}
2 s \\
j
\end{array}\right]=\frac{1}{(x)_{j}} \sum_{t=0}^{j}(-1)^{t} x^{1 / 2} t(t+1)+t(2 s-j)\left[\begin{array}{l}
j \\
t
\end{array}\right],
$$

we get

$$
\begin{aligned}
r_{j} & =\frac{1}{(x)_{j}} \sum_{s=0}^{k}(-1)^{k-s} x^{k-s}\left[\begin{array}{l}
k \\
s
\end{array}\right]^{\prime} x^{(s-j)^{2}} \sum_{t=0}^{j}(-1)^{t} x^{1 / 2 t(t+1)+t(2 s-j)}\left[\begin{array}{l}
j \\
t
\end{array}\right] \\
& =\frac{x^{j^{2}+k}}{(x)_{j}} \sum_{t=0}^{j}(-1)^{t} x^{1 / 2 t(t+1)-t j}\left[\begin{array}{l}
j \\
t
\end{array}\right] \sum_{s=0}^{k}(-1)^{k-s} x^{s^{2}-s}\left[\begin{array}{l}
k \\
s
\end{array}\right]^{\prime} x^{-2 s(j-t)} \\
& =(-1)^{k} \frac{x^{j^{2}+k}}{(x)_{j}} \sum_{t=0}^{j}(-1)^{t} x^{1 / 2 t(t+1)-t j}\left[\begin{array}{l}
j \\
t
\end{array}\right]\left(x^{-2(j-t)}\right)_{k}^{\prime} .
\end{aligned}
$$

Since

$$
\left(x^{-2 t}\right)_{k}^{\prime}= \begin{cases}0 & (0 \leqslant t<k) \\ (-1)^{k} x^{-k(k+1)}\left(x^{2}\right)_{k}^{\prime} & (t=k)\end{cases}
$$

it follows that $r_{j}=0$ for $0 \leqslant j<k$, while

$$
r_{k}=\left(x^{2}\right)_{k}^{\prime} /(x)_{k}=(1+x)\left(1+x^{2}\right) \cdots\left(1+x^{k}\right) \text {. }
$$

Hence

$$
D_{k}=(1+x)\left(1+x^{2}\right) \cdots\left(1+x^{k}\right) D_{k-1} .
$$

Since 


$$
D_{1}=\left|\left[\begin{array}{l}
2 \\
1
\end{array}\right]\right|=1+x
$$

we get

$$
D_{k}=(1+x)^{k}\left(1+x^{2}\right)^{k-1} \cdots\left(1+x^{k}\right) .
$$

Substitution from (6.9) in (6.8) yields

THEOREM 4. We have

$$
Q_{n}(x)=\frac{1}{(1-x)^{n}\left(1-x^{3}\right)^{n-1} \cdots\left(1-x^{2 n-1}\right)}
$$

This completes the proof of (1.11).

7. Number of Maximal Proper Chains in $L_{n}$. As an application of Theorem 4 we have the following.

THEOREM 5. The number of maximal proper chains in $L_{n}$ is given by

$$
M_{n}=\frac{(1 / 2 n(n+1)) !}{1^{n} 3^{n-1} 5^{n-2} \cdots(2 n-1)} .
$$

Proof. By Theorem 4 we see that

$$
\lim _{x \rightarrow 1}(1-x)^{1 / 2 n(n+1)} Q_{n}(x)=\left(1^{n} 3^{n-1} 5^{n-2} \cdots(2 n-1)\right)^{-1} .
$$

On the other hand, by (5.8),

$$
\lim _{x \rightarrow 1}(1-x)^{1 / 2 n(n+1)} Q_{n}(x)=\frac{M_{n}}{(1 / 2 n(n+1)) !} .
$$

Comparison of (7.2) and (7.3) yields (7.1).

8. A Related Partition Problem. Let $T_{k}^{\prime}(n)$ denote the number of triangular arrays $\left(a_{i j}\right)(1 \leqslant j \leqslant i \leqslant k)$ satisfying the inequalities $a_{i j} \geqslant a_{i+1, j}, a_{i j} \geqslant a_{i+1, j+1}$ and also

It can be shown that

$$
\sum_{i=1}^{k} \sum_{j=1}^{i} a_{i j}=n
$$

$$
\sum_{n=0}^{\infty} T_{k}^{\prime}(n) x^{n}=\frac{(x)_{1}(x)_{2} \cdots(x)_{k}}{(x)_{2}(x)_{4} \cdots(x)_{2 k}} D_{k}^{\prime},
$$

where

$$
D_{k}^{\prime}=\left|x^{1 / 2(i-j)(i-j-1)}\left[\begin{array}{r}
2 i \\
j
\end{array}\right]\right| \quad(i, j=1,2, \cdots, k) .
$$

The first few values of $D_{k}^{\prime}$ follow: 


$$
\begin{aligned}
& D_{1}^{\prime}=1+x, \quad D_{2}^{\prime}=(1+x)\left(1+x^{2}\right)^{2} \\
& D_{3}^{\prime}=(1+x)\left(1+x^{2}\right)\left(1+x^{3}\right)\left(1+x^{2}+x^{3}+2 x^{4}+x^{5}+x^{6}+x^{8}\right) .
\end{aligned}
$$

We remark that, when $k \rightarrow \infty$, the generating function (8.1) reduces to the generating function for plane partitions.

\section{Mathematics Department}

Duke University

Durham, North Carolina 27706

1. R. ALTER, "Some remarks and results on Catalan numbers," Proceedings of the Second Louisiana Conference on Combinatorics, Graph Theory and Computing, Baton Rouge, La., 1971, pp. 109-132.

2. L. CARLITZ, "Rectangular arrays and plane partitions," Acta Arith., v. 13, 1967/68, pp. 29-47. MR 36 \#2512.

3. L. CARLITZ, “Sequences, paths, ballot numbers," Fibonacci Quart., v. 10, 1972, no. 5, pp. 531-549. MR 47 \#6498.

4. P. A. M. MACMAHON, Combinatory Analysis. Vol. 2, Cambridge, 1916. 